\title{
Vlastimil Pták
}

Norms and the spectral radius of matrices

Czechoslovak Mathematical Journal, Vol. 12 (1962), No. 4, 555-557

Persistent URL: http://dml.cz/dmlcz/100539

\section{Terms of use:}

(C) Institute of Mathematics AS CR, 1962

Institute of Mathematics of the Czech Academy of Sciences provides access to digitized documents strictly for personal use. Each copy of any part of this document must contain these Terms of use.

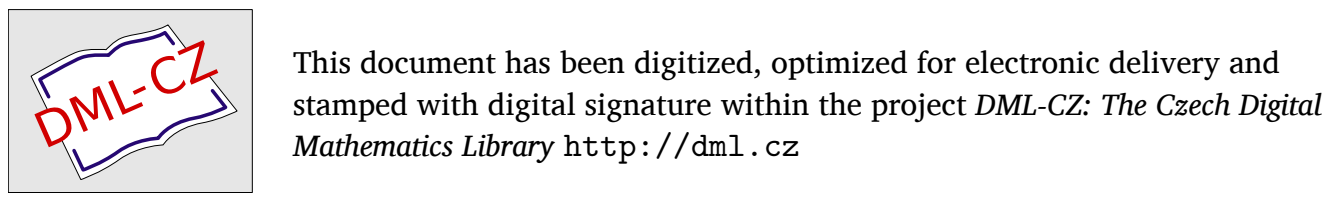




\title{
NORMS AND THE SPECTRAL RADIUS OF MATRICES
}

\author{
Vlastimil Pták, Praha \\ (Received October 25, 1960)
}

\begin{abstract}
The following theorem is proved: If $A$ is a linear operator on an n-dimensional Hilbert space such that $|A|=1$ and also $\left|A^{n}\right|=1$, then $A$ has a proper value $\lambda$ with $|\lambda|=1$. This theorem, together with a simple remark, yield the fact that the critical exponent of a finite-dimensional Hilbert space equals its dimension.
\end{abstract}

1. Let $E$ be a finite-dimensional vector space and $A$ a linear operator in $E$. Consider the equation $x=A x+y$ and the iterative process $x_{r+1}=A x_{r}+y$. It is easy to see that this procedure is convergent for each initial vector $x_{0}$ and each $y$ if and only if the series $E+A+A^{2}+\ldots$ is convergent. The convergence properties of this series are described in the following well-known result.

$(1,1)$ Let $E$ be a finite-dimensional vector space over the real or complex field and let $A$ be a linear operator in $E$. Then the following conditions are equivalent:

(1) the series $E+A+A^{2}+\ldots$ converges,

(2) the powers $A^{r}$ converge to the zero operator,

(3) $\sigma(A)<1$.

Here $\sigma(A)$, the spectral radius of $A$, is defined as the maximum of all $|\lambda|$ where $\lambda$ runs over the proper values of $A$.

Suppose now that a norm $|x|$ is defined on $E$ and let $|T|$ be the operator norm generated by the norm $|x|$. There is a simple connection between the convergence of the series $E+A+A^{2}+\ldots$ and the norms of the powers of $A$. It is not difficult to see that the series is convergent if $\left|A^{p}\right|<1$ for a suitable $p$. On the other hand, if the series converges, we have $\left|A^{r}\right| \rightarrow 0$ so that $\left|A^{p}\right|<1$ for large $p$. It follows that the series $E+A+A^{2}+\ldots$ converges if and only if $\left|A^{p}\right|<1$ for some $p$.

We are thus led to the following problem: Consider a matrix $A$ of norm 1 and construct the sequence $|A|,\left|A^{2}\right|, \ldots$. Clearly $|A| \geqq\left|A^{2}\right| \geqq\left|A^{3}\right| \geqq \ldots$ so that the following two cases are possible: (1) either $\left|A^{r}\right|=1$ for all $r$ so that $\sigma(A)=1$ (2) or $\left|A^{r}\right| \rightarrow 0$ and $\sigma(A)<1$. It is thus natural to ask how far it is necessary to go in this sequence to decide which of the two preceding cases takes place. This leads to the following

$(\mathbf{1 , 2})$ Definition. The number $q$ is said to be the critical exponent of the space $(E,|x|)$ if the following conditions are satisfied:

(1) if $T$ is a linear operator on $E$ and $|T|=\left|T^{q}\right|=1$ then $\sigma(T)=1$.

(2) there exists a linear operator $B$ on $E$ such that $|B|=\left|B^{q-1}\right|=1$ and $\sigma(B)<1$. 
Recently J. MAŘ́́K and the author have found the critical exponent for the $n$-dimensional complex space with the norm $|x|=\max \left|x_{i}\right|$. It equals $n^{2}-n+1$. In the present remark we show that for the $n$-dimensional Hilbert space (norm $|x|=$ $\left.=\left(\Sigma\left|x_{i}\right|^{2}\right)^{1 / 2}\right)$ the critical exponent is $n$.

In the rest. of this remark, $E$ is an $n$-dimensional complex Hilbert space with norm $|x|$ and scalar product $(x, y)$. We shall need two simple lemmas.

$(1,3)$ Let $E$ be a Hilbert space, $A$ a linear operator in $E$ with $|A| \leqq 1$. Let $x_{1}, x_{2}$, $y_{1}, y_{2}$ be vectors of norm 1 such that $y_{i}=A x_{i}$ for $i=1,2$. Then $\left(y_{1}, y_{2}\right)=\left(x_{1}, x_{2}\right)$.

Proof. Let $\alpha_{1}, \alpha_{2}$ be two arbitrary complex numbers. We have the inequality

$$
\left|\alpha_{1} y_{1}+\alpha_{2} y_{2}\right|=\left|A\left(\alpha_{1} x_{1}+\alpha_{2} x_{2}\right)\right| \leqq\left|\alpha_{1} x_{1}+\alpha_{2} x_{2}\right|
$$

and the formula

$$
\begin{gathered}
\left(\alpha_{1} y_{1}+\alpha_{2} y_{2}, \alpha_{1} y_{1}+\alpha_{2} y_{2}\right)= \\
=\left|\alpha_{1}\right|^{2}+\left|\alpha_{2}\right|^{2}+\alpha_{1} \bar{\alpha}_{2}\left(y_{1}, y_{2}\right)+\alpha_{2} \bar{\alpha}_{1}\left(y_{2}, y_{1}\right) ;
\end{gathered}
$$

a similar formula holds for $x_{1}$ and $x_{2}$. It follows that

$$
\xi\left(y_{1}, y_{2}\right)+\bar{\xi}\left(y_{2}, y_{1}\right) \leqq \xi\left(x_{1}, x_{2}\right)+\bar{\xi}\left(x_{2}, x_{1}\right)
$$

for every complex $\xi$. Put $\left(x_{1}, x_{2}\right)=\alpha+i \beta$ and $\left(y_{1}, y_{2}\right)=\sigma+i \tau$ with real $\alpha, \beta$, $\sigma, \tau$. Write down the preceding inequality for $\xi=1,-1, i,-\mathrm{i}$. We obtain $\sigma \leqq \alpha$, $-\sigma \leqq-\alpha,-\tau \leqq-\beta$ and $\tau \leqq \beta$ so that $\left(y_{1}, y_{2}\right)=\left(x_{1}, x_{2}\right)$.

$(1,4)$ Let $E$ be a $t$-dimensional Hilbert space, $A$ a linear operator in $E$ with norm $|A| \leqq 1$. Suppose that there exist $t$ linearly independent vectors $y_{1}, \ldots, y_{t}$ such such that $\left|y_{i}\right|=1$ and $y_{i}=A x_{i}$ for some $x_{i}$ with $\left|x_{i}\right|=1$. Then $A$ is unitary.

Proof. We are going to show that $|A x|=|x|$ for each $x \in E$. To see that, take an $x \in E$; the $y_{i}$ being linearly independent, the $x_{i}$ are linearly independent as well so that $x$ may be expressed in the form $x=\alpha_{1} x_{1}+\ldots+\alpha_{t} x_{t}$. Since $\left|x_{i}\right|=\left|y_{i}\right|=1$, we have $\left(y_{i}, y_{j}\right)=\left(x_{i}, x_{j}\right)$ for each pair of indices according to the preceding lemma. It follows that

$$
\begin{gathered}
(A x, A x)=\left(\sum_{i} \alpha_{i} y_{i}, \sum_{i} \alpha_{i} y_{i}\right)=\sum_{i, j} \alpha_{i} \bar{\alpha}_{j}\left(y_{i}, y_{j}\right)= \\
=\sum_{i, j} \alpha_{i} \bar{\alpha}_{j}\left(x_{i}, x_{j}\right)=\left(\sum_{i} \alpha_{i} x_{i}, \sum_{i} \alpha_{i} x_{i}\right)=(x, x)
\end{gathered}
$$

and the lemma is established.

2. The critical exponent. We are now able to formulate the main result.

$(2,1)$ Theorem. Let $E$ be an $n$-dimensional Hilbert space, $A$ a linear operator of norm 1 in $E$. If $\left|A^{n}\right|=1$, then the spectral radius of $A$ equals 1.

Proof. Let us denote by $V$ the set of all $y \in E$ such that $y=A x$ and $|y|=|x|$ for a suitable $x$. Let $k$ be the maximal number of linearly independent vectors in $V$, so that $1 \leqq k \leqq n$. If $k=n$, the operator $A$ is unitary according to $(1,4)$ so that $\sigma(A)=1$. If $k<n$, take some $k$ linearly independent vectors $y_{1}, \ldots, y_{k} \in V$ and denote by $W$ the linear subspace of $E$ spanned by $y_{1}, \ldots, y_{k}$. It follows from the maximality of $k$ that $V \subset W$. 
Since $\left|A^{n}\right|=1$, there exists a vector $x_{0}$ such that $\left|x_{0}\right|=\left|A^{n} x_{0}\right|=1$. Put $z_{i}=A^{i} x_{0}$ for $i=1,2, \ldots, n$. Clearly $z_{1}, \ldots, z_{n}$ belong to $V$ so that $z_{1}, z_{2}, \ldots, z_{n} \in W$. The dimension of $W$ being $k<n$, it follows that $z_{1}, \ldots, z_{n}$ cannot be linearly independent. Let $z_{q}$ be the first of the $z_{j}$ which may be expressed as a linear combination of the preceding ones, $z_{q}=\alpha_{1} z_{1}+\ldots+\alpha_{q-1} z_{q-1}$.

The vectors $z_{1}, \ldots, z_{q-1}$ are linearly independent because of the minimality of $q$. Take now a $p<q$ such that $\alpha_{p} \neq 0$ and let us show that the vectors $z_{p+1}, \ldots, z_{q}$ are linearly independent as well. To see that, suppose that there is a relation $\beta_{p+1}, z_{p+1}+\ldots+\beta_{q} z_{q}=0$ with at least one $\beta$ different from zero; it follows from the minimality of $q$ that $\beta_{q} \neq 0$ and $p+1<q$. We obtain thus a relation

$$
z_{q}=\gamma_{p+1} z_{p+1}+\ldots+\gamma_{q-1} z_{q-1}=\alpha_{1} z_{1}+\ldots+\alpha_{q-1} z_{q-1}
$$

which is a contradiction since $\alpha_{p} \neq 0$ and $z_{1}, \ldots, z_{q-1}$ are linearly independent.

Now let $p$ be the smallest index with $\alpha_{p} \neq 0$ and let us denote by $H$ the $(q-p)$ dimensional subspace spanned by $z_{p}, \ldots, z_{q-1}$. The space $A(H)$ being generated by $z_{p+1}, \ldots, z_{q}$, we have $A(H) \subset H$ so that we may consider the partial operator $A_{H}$ restricted to $H$. Now there are $q-p$ linearly independent vectors $z_{p+1}, \ldots, z_{q}$ in $H$ such that $\left|z_{i}\right|=1$ and $z_{i}=A x_{i}$ for some $x_{i} \in H$ with $\left|x_{i}\right|=1$. Indeed, it is sufficient to take $x_{i}=z_{i-1}$. It follows from lemma $(1,4)$ that $A_{H}$ is an unitary operator on $H$. Clearly $1=\sigma\left(A_{H}\right) \leqq \sigma(A) \leqq 1$ whence $\sigma(A)=1$ and the theorem is established.

The preceding theorem shows that the critical exponent for $n$-dimensional Hilbert space is at most $n$. The following simple example shows that it is exactly $n$.

$(\mathbf{2 , 2})$ Let $E$ be an $n$-dimensional Hilbert space; then there exists a linear operator $A$ on $E$ such that $|A|=\left|A^{n-1}\right|=1$ and $\sigma(A)=0$.

Proof. Let $e_{1}, \ldots, e_{n}$ be an orthonormal system in $E$ and let $A$ be defined by the relations $A e_{i}=e_{i+1}$ for $i=1,2, \ldots, n-1$ and $A e_{n}=0$. Clearly $|A| \leqq 1$. Since $A^{n-1} e_{1}=e_{n}$, we have $\left|A^{n-1}\right|=1$. At the same time $A^{n}=0$ so that $\sigma(A)=0$.

\section{Bibliography}

[1] J. Mařik, V. Pták: Norms, spectra and combinatorial properties of matrices. Czech. Math. Journal 85 (1960), $181-196$.

\section{Резюме}

\section{НОРМЫ И СПЕКТРАЛЬНЫЙ РАДИУС МАТРИЦ}

\section{ВЛАСТИМИЛ ПТАК (Vlastimil Pták), Прага}

Доказывается следующая теорема: Если $A$ - линейный оператор в $n$-мерном пространстве Гильберта такой, что $|A|=1$ а также $\left|A^{n}\right|=1$, то существует собственное значение $\lambda$ матрицы $A$ с абсолютной величиной 1. Эта теорема вместе с простым построением некоторой матрицы дает тот результат, что критический показатель конечномерного пространства Гильберта равен его размерности. 